\title{
Observational Signatures of Gravitational Couplings in DBI Inflation
}

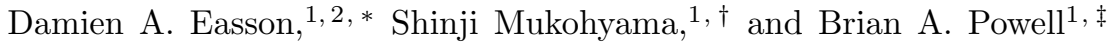 \\ ${ }^{1}$ Institute for the Physics and Mathematics of the Universe, \\ University of Tokyo, 5-1-5 Kashiwanoha, Kashiwa, Chiba 277-8568, Japan \\ ${ }^{2}$ Department of Physics, and School of Earth and Space Exploration, \\ and Beyond Center, Arizona State University, Tempe, AZ, 85287-1504, USA
}

(Dated: September 4, 2021)

\begin{abstract}
In scalar-tensor theories the scalar fields generically couple nontrivially to gravity. We study the observable properties of inflationary models with non-minimally coupled inflaton and Dirac-BornInfeld (DBI) kinetic term. Within the assumptions of the priors of our Monte-Carlo simulations we find these models can generate new interesting observable signatures. Our discussion focuses on string theory inspired phenomenological models of relativistic D-brane inflation. While successful string theory constructions of ultra-violet DBI brane inflation remain elusive, we show that in suitable regions of the parameter space it is possible to use cosmological observables to probe the non-minimial coupling. Fortunately, the most observationally promising range of parameters include models yielding intermediate levels of non-gaussianity in the range consistent with WMAP 5-year data, and to be constrained further by the Planck satellite.
\end{abstract}

\section{INTRODUCTION}

There is substantial evidence indicating that the early Universe underwent a brief period of rapid, accelerated expansion, or inflation 1 3. In addition to solving conceptual and theoretical problems of the Standard Big Bang model (such as the monopole, flatness and horizon problems), inflation provides a quantum origin for the seeds of large scale structures in the Universe and produces theoretical predictions that are supported by detailed measurements of temperature anisotriopies in the cosmic microwave background radiation (CMB) [4]. But, despite its many phenomenological successes, the microphysical origin of inflation remains unclear; indeed, ever since its inception, it has been a challenge to derive successful inflationary models from fundamental particle physics 5]. Fortunately, early work on D-brane inflation in string theory [6 11, has fruitfully led to promising realizations of the scenario ${ }^{1}$.

Many inflationary models are driven, in part, by an inflaton field with non-canonical kinetic term, such as kinflation [14, Ghost inflation [15] and Dirac-Born-Infeld (DBI) inflation [16]. In these models the speed of sound can be significantly smaller than unity. Because cosmological perturbations travel at speeds less than that of light in these models, distinct observational signals are typically generated, including detectable levels of nonGaussianity, e.g. [16 19. As we demonstrate in this paper, non-Gaussianity is an additional observable providing new information that can be used to distinguish between certain inflationary models.

It is the nature of quantum field theory in curved

\footnotetext{
*Email easson@asu.edu

${ }^{\dagger}$ Email Shinji.mukohyama@ipmu.jp

‡Email:brian.powell@ipmu.jp

${ }^{1}$ For recent reviews see, e.g. $12,13$.
}

spacetime that scalar fields generically couple nonminimally to gravity 20]. In this paper we study effects of gravitational couplings in inflationary models with non-standard kinetic terms. In particular, we are interested in determining how such couplings alter cosmological observables. Our goal is to determine if it is possible to probe such couplings using measurements of non-Gaussianity together with measurements of other standard physical quantities. We ultimately focus on the theoretically well-motivated setting of a non-minimally coupled inflaton field in a DBI inflationary model; however, the methods we develop are applicable to more general settings as discussed below.

An outline of the paper is as follows: In $\$ 2$, we provide a brief introduction to gravitational couplings in theories with non-standard kinetic terms and DBI models of D-brane inflation. In $\$ 3$ we derive analytic expectations for the observables of canonical and non-canonical models with conformal coupling. In $\$ 4$, we discuss the prospects of observationally discriminating between pure DBI theories and DBI theory with a conformal coupling term. In $\$ 5$. we describe the priors and general methodology used in our Monte-Carlo simulations. In $\$ 6$, we present our interpretation and an analytic understanding of the results of the analysis. Finally, we provide a summary of significant findings and further conclusions in 87. Technical calculations are relegated to a series of Appendices.

\section{MOTIVATION}

The most general actions we would like to study are of the form

$$
S=\int d^{4} x \sqrt{-g}(F(R, \varphi)+P(X, \varphi)),
$$

where $X=-\frac{1}{2} g^{\mu \nu} \partial_{\mu} \varphi \partial_{\nu} \varphi$, so that for a canonically normalized scalar field, $P(X, \varphi)=X-V(\varphi) . F$ is a series whose leading contribution is the Einstein-Hilbert term 
and further terms encompass possible gravitational coupling and correction terms ${ }^{2}$ :

$$
F(R, \varphi)=\frac{R}{2} \varphi^{2}\left(M_{\mathrm{P} 1}^{2} \frac{1}{\varphi^{2}}-\xi-\xi_{1} \frac{R}{\varphi^{2}}+\cdots\right)
$$

Gravitational couplings of the form 2 are commonly generated in dynamical 4D gravity, and have been explicitly computed for D-brane probes in curved backgrounds [21, 22], and in D-brane inflationary models [1]. For a recent discussion of the effects of loop corrections on slow-roll inflation models in a power-counting formalism of effective field theory see 23 .

\subsection{DBI Inflation}

From a theoretical perspective, a particularly wellmotivated instance of non-standard kinetic term occurs in D-brane inflation: the inflaton kinetic term is of the Dirac-Born-Infeld (DBI) form [16]:

$$
P(X, \varphi)=-f^{-1}(\varphi) \sqrt{1-2 f(\varphi) X}+f^{-1}(\varphi)-V(\varphi)
$$

where the warp factor $f$ is related to the harmonic function of a warped compactification. The non-standard form of the kinetic Lagrangian imposes an effective speed limit on the inflaton field, analogous to the speed limit imposed on particle motion in Special Relativity. This behavior results in a new form of slow-roll inflation even when the potential is very steep [16, 18.

The most well studied D-brane inflation scenario consists of a brane anti-brane pair embedded in the warped throat region of a conformally Calabi-Yau flux compactification in Type IIB string theory. The warping can be used to obtain a large hierarchy in scales between the electroweak and Planck scales. If the throat is the warped deformed conifold [24, it is well approximated by simple Anti-de-Sitter (AdS) space far from the tip. In the case of ultra-violet (UV) brane inflation, a mobile D3 brane falls from the UV end of the warped throat towards the infra-red (IR) end of the throat where an anti-D3 brane is located. The position of the D3 brane in the extra dimensions acts as an inflaton field as the brane moves toward the anti-brane under the influence of an attractive Coulomb force. Inflation ends via a tachyonic instability when the brane crashes into the anti-brane. The formal construction is now well known and we do not review the details of the model here. The curious reader is referred to the recent reviews [12, 13. The most successful versions of this scenario are slow-roll inflationary models.

\footnotetext{
${ }^{2}$ We work with the reduced Planck mass, written in terms of the four-dimensional Newton's constant $G_{N}$ as, $M_{\mathrm{Pl}}=1 / \sqrt{8 \pi G_{N}} \simeq$ $2.4 \times 10^{18}$.
}

We briefly comment on the difficulties involved in building rigorous models of UV DBI inflation within the above setting.

In the state-of-the-art constructions of D-brane inflation the inflaton field range in Planck units is bounded 25, 26] by

$$
\frac{\Delta \varphi}{M_{\mathrm{Pl}}} \leq \frac{2}{\sqrt{N_{0}}}
$$

where $N_{0} \gg 1$ is an integer representing the flux stabilizing the throat. The relatively few models capable of satisfying this bound and producing observable levels of non-Gaussianity typically predict a blue spectral index and a negligible tensor component [27]. Finally, DBI constructions must overcome difficult backreaction constraints [16, 28, 30].

In this paper we are interested in the general observational properties catalyzed by combining the DBI action with gravitational couplings, and work within a phenomenological setting inspired by the afore mentioned brane inflation scenario. It is our modest goal to ascertain the possibility of observing gravitational couplings in inflationary models with non-standard kinetic terms and not to build realistic particle physics models. While all of the presented solutions conform to the latest observational constraints imposed by the WMAP satellite [4, the majority do not obey the constraint on the field range (4). Interesting models of inflation in string theory capable of avoiding this bound have recently been proposed in 3134 . From the effective field theory standpoint, the DBI action is of particular interest even divorced from a particular string theory model as it represents the minimal realization of non-linear Lorentz invariance.

\subsection{Equations of Motion and Gravitational Coupling}

Variation of the action (1) using (3) with respect to the metric $g^{\mu \nu}$ leads to the generalized Einstein equation:

$$
\begin{array}{r}
F_{R} R_{\mu \nu}-\frac{1}{2} g_{\mu \nu} F+g_{\mu \nu} \square F_{R}-\nabla_{\mu} \nabla_{\nu} F_{R}= \\
\frac{\partial_{\mu} \varphi \partial_{\nu} \varphi-g_{\mu \nu}\left(\partial^{\sigma} \varphi \partial_{\sigma} \varphi+f^{-1}(\varphi)\right)}{\sqrt{1+f(\varphi) g^{\mu \nu} \partial_{\mu} \varphi \partial_{\nu} \varphi}} \\
+\left(f^{-1}(\varphi)-V(\varphi)\right) g_{\mu \nu}
\end{array}
$$

where $F_{R} \equiv \partial F / \partial R$. The equation of motion for the field $\varphi$ is:

$$
\begin{aligned}
& \nabla_{\mu}\left(\gamma \partial^{\mu} \varphi\right)+f^{-2} f^{\prime}\left(\gamma^{-1}-1\right) \\
& \quad-\frac{1}{2} f^{-1} f^{\prime} \gamma g^{\mu \nu} \partial_{\mu} \varphi \partial_{\nu} \varphi-V^{\prime}-F^{\prime}=0
\end{aligned}
$$

where $\prime \equiv \partial / \partial \varphi$, and we have defined

$$
\gamma \equiv \frac{1}{\sqrt{1+f(\varphi) g^{\mu \nu} \partial_{\mu} \varphi \partial_{\nu} \varphi}}
$$


In this paper we focus on a particular well-motivated and simple form for the gravitational coupling and truncate $F(R, \varphi)$ at the second term in $(2)$. This term, $-\frac{1}{2} \xi R \varphi^{2}$, plays a significant role in brane inflation since the field associated to the brane position is a conformally coupled scalar [11, 16, 21]. The term is ubiquitous in effective field theories of quantum fields in curved spacetime [20, and is renormalizable by power counting arguments.

\section{ANALYTIC ANALYSIS}

\subsection{Canonical Models}

The effects of non-minimal couplings of the form $\xi R \varphi^{2}$ were first investigated in the context of canonical inflation, described by the action,

$$
\begin{aligned}
S= & \int d^{4} x \sqrt{-g}\left\{\frac{M_{\mathrm{Pl}}^{2}}{2} R-\frac{\xi}{2} R \varphi^{2}\right. \\
& \left.-\frac{1}{2} g^{\mu \nu} \partial_{\mu} \varphi \partial_{\nu} \varphi-V(\varphi)\right\},
\end{aligned}
$$

in which the kinetic term of the field is canonically normalized, $\dot{\varphi}^{2} / 2$. From (8) we see that introducing the non-minimal coupling term generates an effective Planck mass of the form

$$
M_{e f f} \equiv \sqrt{M_{\mathrm{Pl}}^{2}-\xi \varphi^{2}} .
$$

For field values larger than the critical value,

$$
\varphi_{c}=\frac{M_{\mathrm{Pl}}}{\sqrt{\xi}}
$$

the effective Newton's constant can become negative. ${ }^{3}$ In a cosmological background with non-minimally coupled scalar (and positive $\xi$ ) the anisotropic shear diverges without bound as $\varphi$ approaches $\varphi_{c}$ [35, 36]. We will regard such large field values as outside the range of validity of our effective theory. In all of the following analysis we ensure that $\varphi<\varphi_{c}$.

The observables of such theories are typically computed in the conformally-related Einstein frame, in which the inflaton is minimally coupled to gravity. The Einstein frame action is obtained by performing the well known Weyl rescaling of the metric,

$$
\tilde{g}_{\mu \nu}=\Omega^{2} g_{\mu \nu},
$$

where $\Omega^{2}=1-\xi \frac{\varphi^{2}}{M_{\mathrm{P}}^{2}}$, followed by a field redefinition, $\varphi \rightarrow \sigma$ (see Appendix A) so that the action becomes

\footnotetext{
${ }^{3}$ For the present discussion we will always take $\xi \geq 0$. Subsequently, we will focus on the conformal value $\xi=1 / 6$ (cf. discussion at the end of 4 .
}

$$
S=\int d^{4} x \sqrt{-\tilde{g}}\left\{\frac{M_{\mathrm{Pl}}^{2}}{2} \tilde{R}-\frac{1}{2} \tilde{g}^{\mu \nu} \partial_{\mu} \sigma \partial_{\nu} \sigma-\tilde{V}(\sigma)\right\},
$$

where $\tilde{V}(\sigma)=V(\varphi) \Omega^{-4}(\varphi)$. While this conformally related theory is physically distinct from the original Eq. (8), the cosmological observables calculated in this frame are equivalent [37. Therefore, we conclude that any nonminimally coupled canonical single-field theory is observationally equivalent to some minimally coupled canonical theory (within the range of validity of the transformation (11)). This makes it impossible to observationally determine the nature of the gravitational inflaton coupling in canonical theories. The result is in agreement with the analysis of 38 .

\subsection{Non-Canonical Models}

We next consider the case of a non-canonical inflaton. In what follows, we study DBI inflation [16, 18] as a prototype non-canonical theory. The standard minimally coupled DBI action is

$$
\begin{aligned}
S= & \int d^{4} x \sqrt{-g}\left\{\frac{M_{\mathrm{Pl}}^{2}}{2} R-\right. \\
& \left.\frac{1}{f(\theta)}\left[\sqrt{1+f(\theta) g^{\mu \nu} \partial_{\mu} \theta \partial_{\nu} \theta}-1\right]-V(\theta)\right\} .
\end{aligned}
$$

In brane inflation $f(\theta)$ is the warp factor of the compactified geometry and $V(\theta)$ is the potential that arises from the presence of anti-D3-branes and other perturbative and non-perturbative sources. The action for single field DBI inflation with non-minimal coupling is

$$
\begin{aligned}
S= & \int d^{4} x \sqrt{-g}\left\{\frac{M_{\mathrm{Pl}}^{2}}{2} R-\frac{1}{2} \xi R \phi^{2}-\right. \\
& \left.\frac{1}{f(\phi)}\left[\sqrt{1+f(\phi) g^{\mu \nu} \partial_{\mu} \phi \partial_{\nu} \phi}-1\right]-V(\phi)\right\} .
\end{aligned}
$$

As in the case of the canonical theory, we can remove the explicit nonminimal coupling via the transformation (11). This results in the action

$$
S=\int d^{4} x \sqrt{-\tilde{g}}\left(\frac{M_{\mathrm{Pl}}^{2}}{2} \tilde{R}+P(X, \phi)\right),
$$

where

$$
X=-\frac{1}{2} \tilde{g}^{\mu \nu} \partial_{\mu} \phi \partial_{\nu} \phi
$$

The corresponding functional form for $P$ is:

$$
\begin{aligned}
& \left(1-\xi \frac{\phi^{2}}{M_{\mathrm{Pl}}^{2}}\right)^{2} P(\phi, X)=6 \xi^{2} X \frac{\phi^{2}}{M_{\mathrm{Pl}}^{2}} \\
& -f^{-1}(\phi) \sqrt{1-2 X f\left(1-\xi \frac{\phi^{2}}{M_{\mathrm{Pl}}^{2}}\right)}+f^{-1}(\phi)-V(\phi) .
\end{aligned}
$$


Therefore, in terms of a homogeneous field $\phi(t)$, the Lagrangian (15) becomes:

$$
\begin{aligned}
\frac{\mathcal{L}}{\sqrt{-\tilde{g}}}= & \frac{M_{\mathrm{Pl}}^{2}}{2} \tilde{R}+g(\phi, \dot{\phi})+ \\
& \Omega^{-4}\left[\left(1-\sqrt{1-\dot{\phi}^{2} f(\phi) \Omega^{2}}\right) \frac{1}{f(\phi)}-V(\phi)\right]
\end{aligned}
$$

where

$$
g(\phi, \dot{\phi})=3 \xi^{2} \frac{\dot{\phi}^{2}}{\Omega^{4}} \frac{\phi^{2}}{M_{\mathrm{Pl}}^{2}} .
$$

The presence of the function $g(\phi, \dot{\phi})$ makes it impossible to fully duplicate the observables derived from (14) using observables derived from a standard minimally coupled DBI theory 13 . Because minimally coupled DBI inflation makes specific observational predictions [27, 39, it is possible that this departure from a pure DBI theory might lead to distinct observable physics. As a first step towards exploring this possibility, we examine Eq. 118 under various field redefinitions.

\subsubsection{Non-Relativistic Limit}

We first redefine the scalar field so that its kinetic term reduces to the canonical form in the non-relativistic limit, $f(\phi) \dot{\phi}^{2} \ll 1$. In the non-relativistic limit the action 18 is reduced to

$$
\begin{aligned}
S_{n r} & =\int d^{4} x \sqrt{-\tilde{g}}\left[\frac{M_{\mathrm{Pl}}^{2}}{2} \tilde{R}\right. \\
& \left.-\frac{1-\xi(1-6 \xi) \frac{\phi^{2}}{M_{\mathrm{Pl}}^{2}}}{2\left(1-\xi \frac{\phi^{2}}{M_{\mathrm{Pl}}^{2}}\right)^{2}} \tilde{g}^{\mu \nu} \partial_{\mu} \phi \partial_{\nu} \phi-\frac{V}{\Omega^{4}}\right] .
\end{aligned}
$$

Thus, we define a new field $\Phi_{c}$ by

$$
d \Phi_{c}=\frac{\sqrt{1-\xi(1-6 \xi) \frac{\phi^{2}}{M_{\mathrm{P} 1}^{2}}}}{1-\xi \frac{\phi^{2}}{M_{\mathrm{P} 1}^{2}}} d \phi
$$

so that

$$
S_{n r}=\int d^{4} x \sqrt{-\tilde{g}}\left[\frac{M_{\mathrm{Pl}}^{2}}{2} \tilde{R}-\frac{1}{2} \tilde{g}^{\mu \nu} \partial_{\mu} \Phi_{c} \partial_{\nu} \Phi_{c}-\tilde{V}\left(\Phi_{c}\right)\right],
$$

where $\tilde{V}\left(\Phi_{c}\right) \equiv V(\phi) / \Omega^{4}(\phi)$. Taking $\xi=1 / 6$, we can integrate 21 to obtain the relation,

$$
\frac{\phi}{M_{\mathrm{Pl}}}=\sqrt{6} \tanh \left(\frac{\Phi_{c}}{\sqrt{6} M_{\mathrm{Pl}}}\right) .
$$

Since the minimally coupled DBI theory 13 reduces to the same non-relativistic form as $(22)$, we expect no physical differences between the theories in this limit.

\subsubsection{Ultra-Relativistic Limit}

We next consider a different field redefinition which brings Eq. (18) into physical agreement with minimally coupled DBI inflation when the field is highly relativistic. In this regime, where the expression inside the square root becomes small, the warp factor plays a dominant role in determining the dynamics. This suggests a field redefinition $\phi \rightarrow \Phi$ that brings the expression inside the square root into the form present in minimally coupled DBI, $1-\dot{\Phi}^{2} f(\Phi)$. Thus, we define a new field,

$$
\frac{\sqrt{\xi} \phi}{M_{\mathrm{Pl}}}=\sin \left(\frac{\sqrt{\xi} \Phi}{M_{\mathrm{Pl}}}\right)
$$

so that

$$
\tilde{\mathcal{L}}=\tilde{\mathcal{L}}_{D B I}+g(\Phi, X)
$$

where

$$
\begin{gathered}
\tilde{\mathcal{L}}_{D B I}=\frac{1}{\tilde{f}}[1-\sqrt{1-2 \tilde{f} X}]-\tilde{V}\left(\Phi_{c}\right), \\
g(\Phi, X)=6 \xi X \tan ^{2}\left(\frac{\sqrt{\xi} \Phi}{M_{\mathrm{Pl}}}\right)
\end{gathered}
$$

and

$$
X=-\frac{1}{2} \tilde{g}^{\mu \nu} \partial_{\mu} \Phi \partial_{\nu} \Phi
$$

Here, $\Phi_{c}$ in the potential $\tilde{V}\left(\Phi_{c}\right)$ must be expressed in terms of $\Phi$ by eliminating $\varphi$ from definitions of $\Phi$ and $\Phi_{c}$, i.e. $\tilde{V}\left(\Phi_{c}(\Phi)\right)$. For $\xi=1 / 6$ we have the relation

$$
\tanh \left(\frac{\Phi_{c}}{\sqrt{6} M_{\mathrm{Pl}}}\right)=\sin \left(\frac{\Phi}{\sqrt{6} M_{\mathrm{Pl}}}\right) \text {. }
$$

In the relativistic limit, $2 \tilde{f} X \approx 1$, and since $6 \xi \tan ^{2}\left(\sqrt{\xi} \Phi / M_{\mathrm{Pl}}\right) \ll 1$ for $\xi \sim \mathcal{O}\left(10^{-1}\right)$ and $\Phi \sim$ $\mathcal{O}\left(M_{\mathrm{Pl}}\right)$ (parameter magnitudes of interest in this paper), $g$ is small and Eq. 25) reduces to the minimally coupled DBI Lagrangian (13). We elaborate on this issue in $\S 6$.

\subsubsection{Intermediate Regime}

Since the above two field redefinitions result in a conformal theory that is almost of the same form as a minimally coupled DBI theory in the non-relativistic and relativistic regimes, respectively, we expect both theories to be observationally degenerate in these limits. However, it is unclear what the nature of the theory given by Eq. 118 is in the intermediate regime. Might there be a region of parameter space for which this theory gives different predictions from a minimally coupled DBI theory? If DBI is the presumed inflationary theory, any observational differences between the two theories could yield direct information about the presence of gravitational couplings, in contrast to the case of canonical models. In the next section, we pursue this question in detail. 


\section{OBSERVATIONALLY DISCRIMINATING NONMINIMAL THEORIES}

\subsection{Non-Gaussianity}

Non-canonical inflation models have the defining characteristic that inflaton fluctuations generally propagate at speeds less than that of light. As a result, the fluctuations are non-Gaussian, at levels significantly larger than the negligible levels produced in single field slow-roll inflation [40]. One non-Gaussian statistic is the three-point function of the co-moving curvature perturbation, $\zeta$, or its fourier transform, the bispectrum [41, 42]:

$$
\left\langle\zeta\left(\mathbf{k}_{1}\right) \zeta\left(\mathbf{k}_{2}\right) \zeta\left(\mathbf{k}_{3}\right)\right\rangle .
$$

The bispectrum is characterized by its amplitude, shape, and scale dependence as a function of comoving wavenumbers $k_{1}, k_{2}$, and $k_{3}$. For the leading-order contributions to the non-Gaussianities in general noncanonical theories we define the estimators [19, ${ }^{4}$

$$
\begin{aligned}
f_{N L}^{\lambda} & =-\frac{5}{81}\left(\frac{1}{c_{s}^{2}}-1-2 \Lambda\right), \\
f_{N L}^{c} & =\frac{35}{108}\left(\frac{1}{c_{s}^{2}}-1\right),
\end{aligned}
$$

where

$$
\Lambda=\frac{X P_{, X X}+\frac{2}{3} X^{2} P_{, X X X}}{P_{, X}+2 X P_{, X X}}
$$

with $P$ the fluid pressure. The hydrodynamical sound speed is defined

$$
c_{s}^{2}=\frac{P_{, X}}{P_{, X}+2 X P_{, X X}} .
$$

These two contributions correspond to different shapes in Fourier space [19, 43. In the case of DBI inflation, $f_{N L}^{\lambda}$ is identically zero, and so an experimental detection of the shape of the non-Gaussianities offers the possibility of distinguishing DBI inflation from more general non-canonical scenarios, in particular, its nonminimally coupled counterpart. However, both shapes are maximized for equilateral configurations $\left(k_{1}=k_{2}=k_{3}\right)$ and will be difficult to distinguish in practice unless there is a detection of high significance 44. We will therefore not consider using the shape of non-Gaussianities as a discriminator. Nevertheless, in [45, it was suggested that since the speed of sound in the non-minimal DBI models differs from that of pure DBI, it may be possible to distinguish between the two theories through cosmological observables.

\footnotetext{
${ }^{4}$ Our sign conventions for $f_{N L}$ are opposite to those of the WMAP team.
}

\subsection{Observables}

We examine the full set of observables for each model, including the amplitude of non-Gaussianities as well as power spectra. We model the power spectrum as a powerlaw + running,

$$
P_{\mathcal{R}}(k)=A_{s}\left(\frac{k}{k_{0}}\right)^{1-n_{s}+\frac{1}{2} \alpha \ln \left(\frac{k}{k_{0}}\right)},
$$

and include a tensor contribution. We adopt a Monte Carlo approach [27, 46, 47] - we stochastically generate large numbers of inflation models of both types (minimal and nonminimal) and calculate their observables, $\left(n_{s}, \alpha, r, f_{N L}\right)$. We then compare the distribution of predictions of each theory in the observable parameter space, and identify regions that are populated by one model and not the other. One can then discuss the plausibility of distinguishing these models with future experiments.

General non-canonical inflation leads to a power spectrum of primordial curvature perturbations of amplitude [17,

$$
P_{\mathcal{R}}(k)=\frac{1}{8 \pi^{2} M_{\mathrm{Pl}}^{2}} \frac{H^{2}}{c_{s} \epsilon},
$$

and a spectrum of gravitational waves of amplitude,

$$
P_{h}(k)=\frac{2}{\pi^{2}} \frac{H^{2}}{M_{\mathrm{Pl}}^{2}} .
$$

Here, $c_{s}$ is as defined in Eq. (34), and

$$
\epsilon=\frac{X P_{, X}}{M_{\mathrm{Pl}}^{2} H^{2}} .
$$

Because the density perturbations travel at speed $c_{s}$, Eq. (36) is to be evaluated at sound horizon crossing, $c_{s} k=$ $a H$, for each comoving wavenumber $k$. However, because the tensor perturbations propagate at the speed of light, Eq. (37) is evaluated when $k=a H$, with the result that scalar and tensor perturbations on the same comoving scale $k$ exit the horizon at different times. Taking this difference into account can be important [48 51]. For a given scale $k$, the tensor-to-scalar ratio

$$
r \equiv \frac{P_{h}}{P_{\mathcal{R}}},
$$

can be evaluated at any time between this mode's scalar crossing and tensor crossing, since the quantities in it are assumed constant over this time period. For the solutions studied in this analysis, we have verified that the difference in horizon crossings is not important $\left(H_{t} \approx H_{s}\right)$. In this case we may use the familiar

$$
r=16 \epsilon c_{s} .
$$

Additionally, we define the scalar spectral index,

$$
n_{s}-1=\frac{d \ln P_{\mathcal{R}}(k)}{d \ln k}=-2 \epsilon-\eta-s,
$$



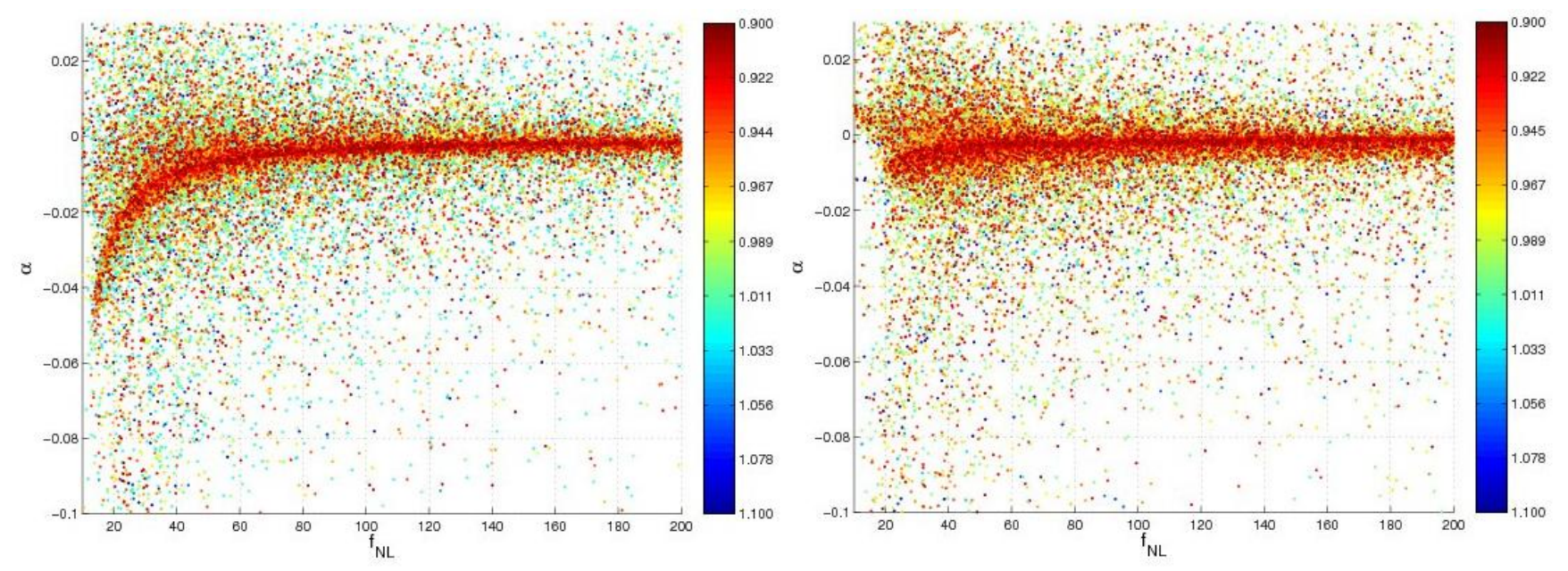

FIG. 1: Monte Carlo results for 60,000 models each of DBI with conformal coupling (left) and minimal coupling (right). Coloring denotes the value of $n_{s}$. Observables are quoted at $k=0.002 \mathrm{hMpc}^{-1}$.

where

$$
\begin{aligned}
\eta & =\frac{\dot{\epsilon}}{\epsilon H}, \\
s & =\frac{\dot{c_{s}}}{c_{s} H} .
\end{aligned}
$$

The running of the spectral index, $\alpha=\frac{d n_{s}}{d \ln k}$, can be obtained from the above via the relation,

$$
\frac{d \ln k}{d \phi}=M_{\mathrm{Pl}} \frac{\sqrt{2 \epsilon}}{\epsilon-1} .
$$

In the conventions we are using, the action (8) (without potential), is conformally invariant for the special value $\xi=\frac{1}{6}$. The presence of a general potential or a nonstandard kinetic term (such as in DBI) will break this symmetry. When it is necessary for us to consider an actual numerical value for the non-minimal coupling parameter, we shall take it to be $\xi=\frac{1}{6}$. We may also casually refer to the case of non-minimal coupling as the conformal case.

\section{MONTE-CARLO ANALYSIS}

\subsection{Equations of Motion}

We obtain equations of motion for the minimal models from the Lagrangian (13),

$$
\begin{aligned}
3 M_{\mathrm{Pl}}^{2} H^{2} & =\rho \\
& =f^{-1}(\gamma-1)+V, \\
2 M_{\mathrm{Pl}}^{2} \dot{H} & =-(\rho+P) \\
& =\gamma f^{-1}\left(\gamma^{-2}-1\right) .
\end{aligned}
$$

The equations of motion for the conformal models are obtained from Eq. 25,

$$
\begin{aligned}
3 M_{\mathrm{Pl}}^{2} \tilde{H}^{2} & =\tilde{\rho} \\
& =\tilde{f}^{-1}(\tilde{\gamma}-1)+g_{, X} X+\tilde{V}, \\
2 M_{\mathrm{P} 1}^{2} \dot{\tilde{H}} & =-(\tilde{\rho}+\tilde{P}) \\
& =-(\gamma+g) X .
\end{aligned}
$$

where $\tilde{\gamma}=(1-2 \tilde{f} X)^{-1 / 2}$.

\subsection{Priors on $V$ and $f$}

Care should be taken to appropriately determine the priors on the functions $V(\theta), \tilde{V}(\Phi)$, and $f(\theta), \tilde{f}(\Phi)$. We must ensure that any observable differences found between these models is truly a result of physics and not a poor choice of priors for these functions. Note that Eqs. 45 are equivalent to Eqs. 46 when $g=0$, $V(\theta) \simeq V(\Phi)$, and $f(\theta) \simeq \tilde{f}(\Phi)$. This is because the Lagrangian Eq. 26 coincides with that of minimally coupled DBI in the relativistic limit, when the warp factor, $f$, is important. Therefore, to minimize physical differences, we should choose the same priors for $f(\theta)$ and $\tilde{f}(\Phi)$. However, Eq. 26 is not equivalent to the minimally coupled Lagrangian in the non-relativistic limit, when $V$ is important. Therefore, we cannot impose the prior $V(\theta) \leftrightarrow \tilde{V}(\Phi)$. Rather, we must appeal to the Lagrangian 20, which does coincide with minimally coupled DBI in the non-relativistic model. This suggests the prior $V(\theta) \leftrightarrow \tilde{V}\left(\Phi_{c}\right)$.

\subsection{Parameter Values and Methodology}

With the proper priors in place, we now define the functional forms of the potentials and the warp factors. 
For the potentials we consider the Taylor expansion,

$$
\begin{gathered}
V(\theta)=\sum_{n=0}^{\infty} \frac{V_{n}}{2 n !}\left(\frac{\theta}{M_{\mathrm{Pl}}}\right)^{n}, \\
\tilde{V}\left(\Phi_{c}\right)=\sum_{n=0}^{\infty} \frac{\tilde{V}_{n}}{2 n !}\left(\frac{\Phi_{c}}{M_{\mathrm{Pl}}}\right)^{n},
\end{gathered}
$$

truncated at $n=4$. Fourth order is about the extent to which we ever expect to reliably reconstruct the inflaton potential, and hence this form is motivated by phenomenology. We assume that the warp factors are of the AdS form, $f(\theta)=\lambda / \theta^{4}, \tilde{f}(\Phi)=\tilde{\lambda} / \Phi^{4}$. We draw the potential parameters from the ranges $\left|V_{n} / V_{0}\right|$, $\left|\tilde{V}_{n} / \tilde{V}_{0}\right| \in\left[10^{-5}, 10^{5}\right]$, where $V_{0}, \tilde{V}_{0}$ is determined by normalizing the spectra to the best-fit WMAP5 value, $A_{s}=3.2 \times 10^{-9}$ [4. The warp factor is determined by drawing the rescaled warping from the range $\lambda / V_{0}$, $\tilde{\lambda} / \tilde{V}_{0} \in\left[10^{-5}, 10^{3}\right]$. We consider random initial conditions for $\dot{\theta}_{i}$ (and $\dot{\Phi}_{i}$ ) as well as the initial field values, $\theta_{i}$ (and $\Phi_{i}$ ). While the minimally coupled field $\theta$ is unbounded, the conformally coupled field must satisfy $\Phi_{i}<\sqrt{\frac{3}{2}} \pi M_{\mathrm{Pl}}$, corresponding to the bound $\sqrt{10}$. The Monte Carlo analysis is carried out for the minimal models by numerically solving Eqs (45), and for the conformal models by solving Eqs. 46.

Each solution is evolved forward in time until sufficient inflation is obtained, here chosen to be $N=55 \mathrm{e}^{-}$ foldings. The number of e-foldings is obtained by solving the equation

$$
d N=-H d t
$$

along with each system. Models which yield insufficient inflation are rejected, as are those that evolve to a metastable minimum and eternally inflate. For solutions that reach $N=55$, by cutting off the evolution at this point we are supposing that inflation ends via tachyonic instability in some auxiliary field.

The correspondence between the comoving scale, $k$, and the number of efolds of inflation, $N$, is different for the scalar and tensor modes because of the difference in horizon crossing times. It is determined by solving

$$
\frac{d(a H)}{d t}=(1-\epsilon) a H^{2},
$$

with $k=a H$ for tensors and $k c_{s}=a H$ for scalars. However, as mentioned, the difference in crossing times can be neglected, and so we evaluate all observables at $a H=0.002 h \mathrm{Mpc}^{-1}$.

\section{RESULTS}

We present the results of the Monte Carlo in Fig. 1 for models that lie within approximate WMAP5 95\%
CL (with running and tensors) [4]: $-0.1<\alpha<0.03$, $0.9<n_{s}<1.1,-253<f_{N L}^{\text {equil }}<151^{5}$, and $0<r<0.3$. We depict the $f_{N L^{-}} \alpha$ plane and indicate the value of $n_{s}$ with the color bar, for the conformal model (left panel) and the minimal model (right panel). There is a clear difference in the clustering of points between the two models. For the conformal model, we note the high density of points with red spectral index $\left(n_{s}<1\right)$ with negative running $(-0.1 \lesssim \alpha \lesssim 0)$ and moderate degree of nonGaussianitiy $\left(1 \lesssim f_{N L} \lesssim 60\right)$. If there are observable differences between the theories, it is in this intermediate range that we expect the difference to be present, as the priors are chosen to ensure that the physics is the same in the non-relativistic (low $f_{N L}$ ) and the relativistic (high $\left.f_{N L}\right)$ limits. The lowest limit in this range compatible with the sensitivity expected from Planck for equilateral type non-Gaussianity is $f_{N L}^{\text {equil }} \gtrsim 10$ [54]. We will demonstrate that the most significant differences in models can be seen in the approximate range $f_{N L} \in(20,40)$.

In order to demonstrate the accuracy of our numerical treatment, we verify that the minimal and conformal models do indeed give the same physics in the DBI and slow roll limits. In the left panel of Fig. 2, we present models in the $n_{s}-r$ plane with a large degree of nonGaussianity: $180<f_{N L}^{\text {equil }}<200$. Conformal models are colored blue and minimal models colored green. The two distributions of points overlap, indicating that the models are observationally degenerate in this range. In the opposite limit, $f_{N L}^{\text {equil }}<1$, we again see agreement, in the right panel of Fig. 2. However, when we focus attention on the intermediate region, $20<f_{N L}^{\text {equil }}<40$, in Fig. 3, we discover the surprising result that the two models yield distinct observational predictions. We find that there is an observable region that is populated by only one of the two classes of models; the conformal models can accommodate a redder spectral index for a given $r$ and $f_{N L}$. Additionally, the width of this strip is of the same order as the 1- $\sigma$ error expected from Planck (gray error bar), suggesting that these models may indeed be resolved in practice. As we now show, the breaking of observable degeneracy is because of the difference in the field value at $N=55$ between the minimal and conformal models.

To begin, consider the following expressions for the

\footnotetext{
${ }^{5}$ A recent analysis finds $-435<f_{N L}^{\text {equil }}<125$ 52.
} 

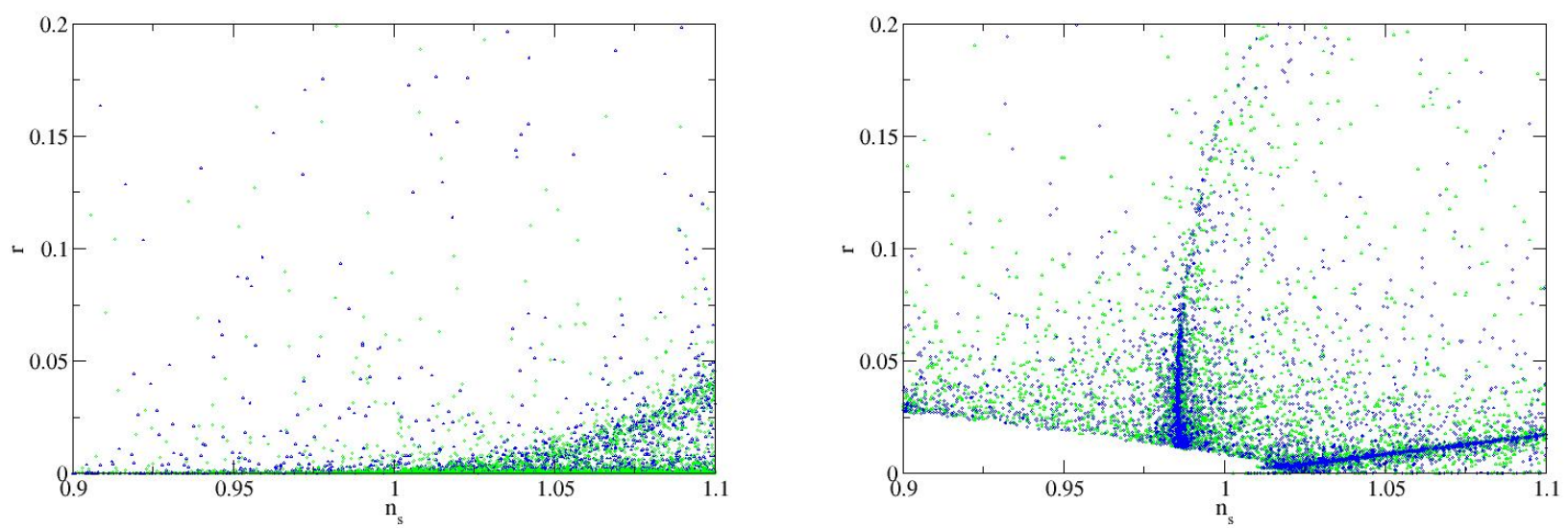

FIG. 2: Monte Carlo results for conformal models (blue) and minimal models (green) satisfying $f_{N L}<1$ (left panel) and $180<f_{N L}<200$ (right panel). There is no detectable difference between the models in these ranges.

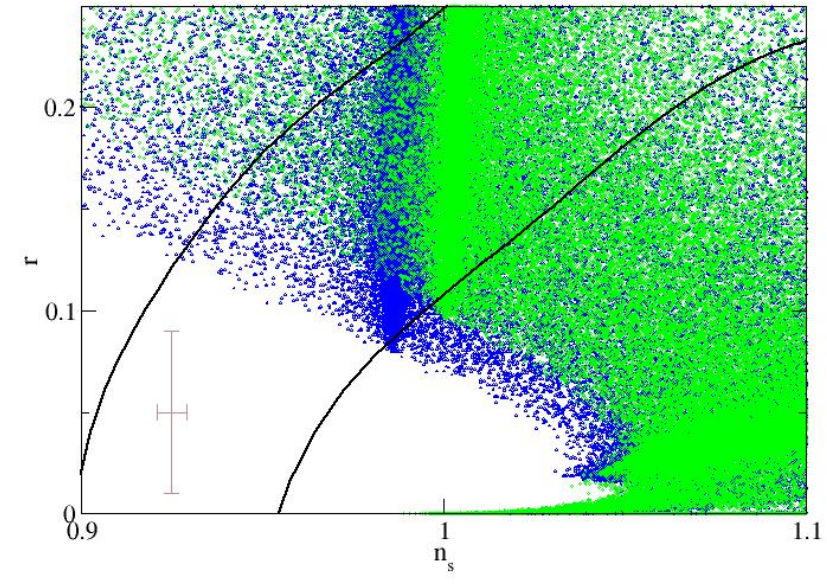

FIG. 3: Monte Carlo results for conformal models (blue) and minimal models (green) satisfying $20<f_{N L}<40$. The contours denote WMAP5+SDSS 1- and 2- $\sigma$ marginalized constraints. The gray error bar is that expected from Planck at $1-\sigma$.
DBI parameters and sound speed,

$$
\begin{aligned}
\epsilon & =-\frac{\dot{H}}{H^{2}}=\frac{\left(\gamma+g_{, X}\right) X}{M_{\mathrm{Pl}}^{2} H^{2}}, \\
\eta & =\frac{\dot{\epsilon}}{H \epsilon}=\frac{1}{H}\left[\frac{\dot{\gamma}+\dot{g}_{, X}}{\gamma+g_{, X}}+\frac{\dot{X}}{X}-\frac{2 \dot{H}}{H}\right] \\
& =\frac{1}{H}\left[\frac{\dot{\gamma}+\dot{g}_{, X}}{\gamma+g_{, X}}+\frac{2 \gamma \dot{\gamma}}{\gamma^{2}-1}-\frac{2 \dot{\gamma}}{\gamma}-\frac{\dot{f}}{f}\right]+2 \epsilon, \\
s & =\frac{\dot{c}_{s}}{H c_{s}}=\frac{1}{2 H}\left[\frac{\dot{\gamma}+\dot{g}_{, X}}{\gamma+g_{, X}}-\frac{3 \gamma^{2} \dot{\gamma}+\dot{g}_{, X}}{\gamma^{3}+g_{, X}}\right], \\
c_{s}^{2} & =\frac{\gamma+g_{, X}}{\gamma^{3}+g_{, X}},
\end{aligned}
$$

where we have taken $\tilde{\gamma} \approx \gamma$. It is now a simple matter to generalize the Lyth bound of [53] (see Appendix C).

Using the expression for the spectral index, Eq. (41), and making various approximations valid for the regions of interest (see Appendix B) we obtain

$$
n_{s}-1 \simeq-\frac{r \sqrt{3 f_{N L}}}{4}+\frac{\sqrt{2 r}}{\chi / M_{\mathrm{Pl}}},
$$

where $\chi$ is a general scalar field denoting either $\theta$ (minimal field) or $\Phi$ (conformal field). This result indicates that the difference seen in Fig. 3 is a result of the difference between $\theta_{55, \max }$ and $\Phi_{55 \text {,max }}$, the maximum field values corresponding to $N=55$. While this is only a rough approximation, it provides a reasonably accurate understanding of Fig. 3. By choosing $f_{N L}=40$ and taking the corresponding values of $\theta_{55 \text {, max }}$ and $\Phi_{55 \text {, max }}$ $\left(\Phi_{55, \max }>\theta_{55, \max }\right)$ in Eq. $(52)$, we obtain the curves approximating the cut-off of each model in the $n_{s}-r$ plane (cf. Fig. 4). In order to justify our choice of constant $f_{N L}$ in this expression, in Fig. 5 we present the same conformal models as in Fig. 3, but with the value of $f_{N L}$ 


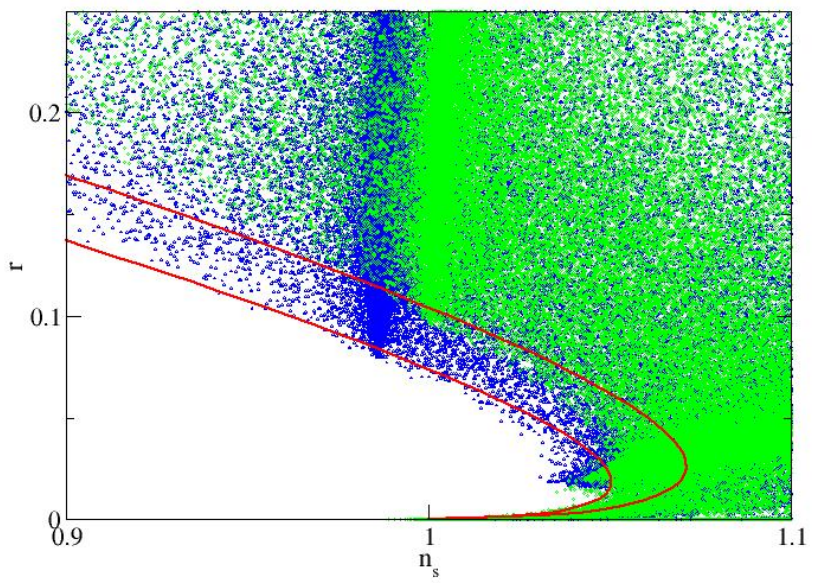

FIG. 4: Same models as in Fig. 3, with theoretical curves obtained from Eq. (52). The upper red curve approximates the cut-off in minimal models, and the lower curve for conformal models.

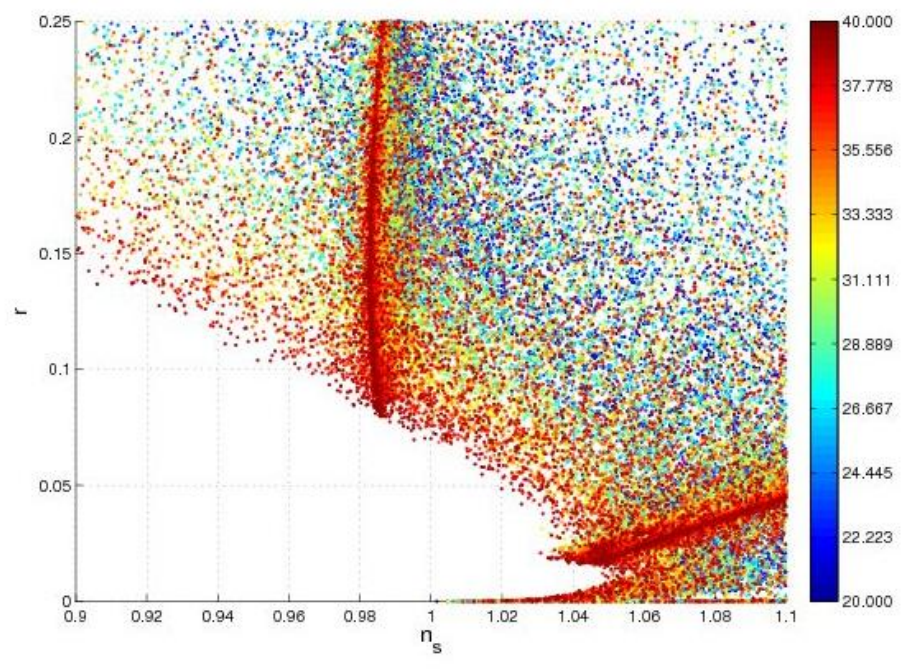

FIG. 5: Same conformal models as in Fig. 3, with the value of $f_{N L}$ color-coded.

color coded. Note that models with the same $f_{N L}$ tend to form bands in the $n_{s}-r$ plane, increasing in magnitude towards small $n_{s}$. These bands are of the same form as the theoretical curves found in Fig. 4.

It remains to understand why $\Phi_{55 \text {, max }}>\theta_{55 \text {, max }}$ in general. In the region under consideration, along the red curves in Fig. 4, we derive the approximate relation

$$
f V \simeq \frac{24}{r}
$$

in Appendix D, This expression is valid for both minimal and conformal models. We compare a minimal model with priors $(f(\theta), V(\theta))$ and a conformal model with

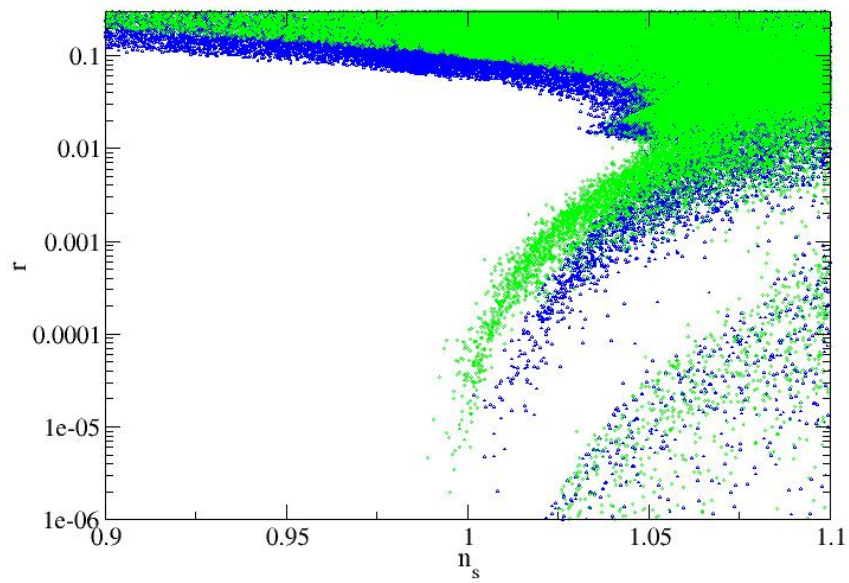

FIG. 6: Close up of the negligible $r$ portion of the parameter space.

$\left(f(\Phi), V\left(\Phi_{c}\right)\right)$. The approximate relation Eq. 53 implies that

$$
f(\Phi) V\left(\Phi_{c}\right) \simeq f(\theta) V(\theta) .
$$

Noting that $V$ is an increasing function, while $f V$ is a decreasing function, $\Phi_{c}>\Phi$ implies that $\Phi$ tends to be larger than $\theta$.

As an illustration, let us consider the case where

$$
f(\theta)=\lambda \theta^{-4}, \quad V(\theta)=V_{0}+V_{4} \theta^{4} .
$$

In this case we have

$$
\theta^{-4} \simeq \Phi^{-4}+\frac{V_{4}}{V_{0}}\left[\left(\frac{\Phi_{c}}{\Phi}\right)^{4}-1\right],
$$

and thus $\Phi>\theta$. Therefore, $\Phi$ tends to be larger than $\theta$. Combining this with the result Eq. (52), we can say that, for $f_{N L}=40$, the minimum value of $n_{s}$ for a given $r$ tends to be smaller for conformal models than for minimal models.

\subsection{Negligible $r$ Regime}

Comparing Fig. 3 and Fig. 5, our attention is drawn to another region of potential interest near the HarrisonZeldovich, $n_{s}=1, r=0$ point. Fig. 6 is a zoom of this region. To investigate this region of the $n_{s}-r$ plane we are required to improve on our estimates of Appendix B. We provide a refined analysis to $\mathcal{O}\left(\gamma^{-1}\right)$ in Appendix $\mathrm{E}$

\subsubsection{Non-Minimally Coupled Models}

We begin with a discussion of the non-minimally coupled (blue) solutions in Fig. 6. We note that there ap- 
pear to be no blue solutions with a red spectral tilt and negligible tensor contribution $r \leq .06$. This is easily understood from equation E3). The last term in E3 is positive since $s$ is negative. This implies that the nonminimal models have blue spectra $\left(n_{s}>1\right)$ in the limit $r \rightarrow 0$. Hence, any detection of $f_{N L}$ in the range $[20,40]$ together with a measure of red spectral index and no detection of $r<.06$ would effectively rule out all nonminimal DBI solutions (within our prior selection).

\subsubsection{Minimally Coupled Models}

We now turn our attention to the minimally coupled DBI models (green solutions). This brings us to a discussion of the large white region in Fig. 6 and the apparent absence of solutions with red spectral index and with low tensor signal $r \leq .06$. As is evident from our large $f_{N L}$ analysis in Fig. 2, allowing models with significantly larger $f_{N L}(>40)$ would gradually fill in this empty region. The only successful red spectrum solutions are a small number of minimally coupled models located near the middle of the Figure. From $(\mathrm{E} 5)$ with $r \approx 0$ we find

$$
n_{s}-1 \simeq \frac{2 s}{3 f_{N L}},
$$

where, in this region we have $s<0$ and $|s|=\mathcal{O}(1)$ and $\theta / M_{\mathrm{Pl}}=\mathcal{O}(1)$. Hence, the reddest the solutions can be are those with $f_{N L}=20$, giving $n_{s} \simeq .97$. The spectrum along the boundary of the populated region turns from blue to red at $r=r_{*}$, where

$$
r_{*} \simeq \frac{1}{2}\left(\frac{2|s| \theta / M_{\mathrm{Pl}}}{3 f_{N L}}\right)^{2} \simeq 10^{-4} .
$$

Hence, any detection of $f_{N L}$ in the range $[20,40]$ together with a measure of red spectral index below $n_{s} \simeq .97$ and no detection of $r<.06$ would effectively rule out all minimally coupled DBI solutions (within our prior selection).

Solutions in the bottom right corner typically have $\chi / M_{\mathrm{Pl}}=\mathcal{O}\left(10^{-2}\right)$, and therefore have a blue spectral tilt since we can no longer ignore the second term in Eqs. (E3), E5.

\section{CONCLUSIONS}

In this paper we studied the effects of a non-minimally coupled inflaton to the DBI action. We assumed an AdS form for the warp factor and studied a wide range of potentials and parameter values using a Monte-Carlo analysis. Our aim was to determine the prospects for observationally distinguishing non-minimal coupling from standard minimally coupled DBI by adopting a phenomenological approach rather than to conform with the latest model building technology in string theory. Our first finding was that:
- There is a strong degeneracy of observables in models with very large and models with undetectable levels of non-Gaussiantiy.

This result is not particularly surprising given our analytic analysis of 3 . However, for an observationally interesting intermediate level of non-Gaussianity with $f_{N L} \in[20,40]$ we find that it is possible, in principle, to distinguish between minimally coupled and nonminimally coupled DBI models. ${ }^{6}$ Assuming a detection of intermediate levels of non-Gaussianity $f_{N L} \in[20,40]$ and the value of the non-minimal coupling parameter $\xi=1 / 6$ we find:

- For a given tensor-to-scalar ratio $r$ and $f_{N L}$, nonminimal DBI models tend to have a redder scalar spectral index $n_{s}$ than minimal DBI models. In other words, for a given scalar spectral index $n_{s}$ and tensor-to-scalar ratio $r, f_{N L}$ tends to be smaller for non-minimal DBI models than for minimal DBI models.

Focusing our attention to the small tensor signal range we find:

- Any detection of a red spectral index $n_{s}$ below .97 combined with no detection of a tensor signal at Planck sensitivity effectively would rule out both non-minimal and minimal DBI inflation models.

- Any detection of a red spectrum and no detection of a tensor signal at Planck sensitivity would rule out non-minimally coupled DBI models.

Finally, the goal of this paper was to initiate the study of observable effects of gravitational couplings in theories with non-standard kinetic terms. While we have focused on the particular example of conformal coupling and DBI kinetic term, our methods are applicable to more general systems represented by the action (1). We leave further study of such models for future research.

\footnotetext{
6 Although our priors are carefully chosen to minimize the differences between the models, our conclusions are dependent upon these priors and the assumptions discussed in detail elsewhere in this paper.
} 


\section{Acknowledgments}

It is a pleasure to thank Ruth Gregory, Simeon Hellerman and Ivonne Zavala for helpful conversations. This work is supported in part by the World Premier International Research Center Initiative (WPI Initiative), MEXT, Japan. DE is also supported by a Grant-in-Aid for Scientific Research (21740167) from the Japan Society for Promotion of Science (JSPS) and by funds from the Arizona State University Foundation. SM is also supported by a Grant-in-Aid for Young Scientists (B) No. 17740134, by JSPS through a Grant-in- Aid for Creative Scientific Research No. 19GS0219, and by the Mitsubishi Foundation.

\section{Appendix A: Conformal Transformation}

Under the transformation

$$
\tilde{g}_{\mu \nu}=\Omega^{2} g_{\mu \nu}
$$

with $\Omega^{2}=1-\xi \frac{\varphi^{2}}{M_{\mathrm{Pl}}^{2}}$, the Ricci scalar transforms as

$$
\tilde{R}=\Omega^{-2}\left[R+3\left(\frac{\Omega^{\prime}}{\Omega}\right)^{2} g^{\mu \nu} \partial_{\mu} \varphi \partial_{\nu} \varphi\right]
$$

where we have omitted a surface term. From Eq. 11, the kinetic term transforms as

$$
-\frac{1}{2} \sqrt{-g} g^{\mu \nu} \partial_{\mu} \varphi \partial_{\nu} \varphi=-\frac{1}{2} \sqrt{-\tilde{g}} \Omega^{-2} \tilde{g}^{\mu \nu} \partial_{\mu} \varphi \partial_{\nu} \varphi
$$

The conformal transformation thus results in a modified kinetic term,

$$
\begin{gathered}
-\frac{1}{2} \sqrt{-\tilde{g}} \mathcal{F} \tilde{g}^{\mu \nu} \partial_{\mu} \varphi \partial_{\nu} \varphi \\
\mathcal{F}=6\left(\frac{\Omega^{\prime}}{\Omega}\right)^{2}+\Omega^{-2}
\end{gathered}
$$

The kinetic term can be made canonical by introducing a new field, $\sigma$, satisfying

$$
\partial_{\mu} \sigma=-\sqrt{\mathcal{F}} \partial_{\mu} \varphi
$$

In terms of this new field, we obtain the Einstein frame action,

$$
S=\int d^{4} x \sqrt{-\tilde{g}}\left\{\frac{M_{\mathrm{Pl}}^{2}}{2} \tilde{R}-\frac{1}{2} \tilde{g}^{\mu \nu} \partial_{\mu} \sigma \partial_{\nu} \sigma-\tilde{V}(\sigma)\right\}
$$

where $\tilde{V}(\sigma)=V(\varphi) \Omega^{-4}(\varphi)$.

\section{Appendix B: Spectral Index}

From the expression for the spectral index, Eq. (41), we obtain

$$
\begin{aligned}
-\left(n_{s}-1\right)-4 \epsilon & =(\eta-2 \epsilon)+s \\
& =\left[-\frac{3 g_{, X}\left(\gamma^{2}-1\right) \gamma}{2\left(\gamma+g_{, X}\right)\left(\gamma^{3}+g_{, X}\right)}\right. \\
& \left.+\frac{2}{\gamma^{2}-1}\right] \frac{\dot{\gamma}}{H \gamma}+\frac{3 \gamma^{3}-\gamma+2 g_{, X}}{2\left(\gamma+g_{, X}\right)\left(\gamma^{3}+g_{, X}\right)} \\
& \times \frac{\dot{g}_{, X}}{H}-\frac{\dot{f}}{H f} \\
& =-\frac{3 g, X \dot{\gamma}}{2 H \gamma^{2}} \times A-\frac{\dot{f}}{H f} \times B,
\end{aligned}
$$

where

$$
\begin{aligned}
A & =\frac{1-\gamma^{-2}}{\left(1+\gamma^{-1} g_{, X}\right)\left(1+\gamma^{-3} g_{, X}\right)}-\frac{4}{3 g_{, X} \gamma} \cdot \frac{1}{1-\gamma^{-2}}, \\
B & =1-\frac{3 g_{, X}}{2 \gamma} \cdot \frac{g_{X}^{\prime} \chi}{g_{, X}} \cdot \frac{f}{f^{\prime} \chi} \cdot \frac{1-\frac{1}{3} \gamma^{-2}+\frac{2}{3} \gamma^{-3} g_{, X}}{\left(1+\gamma^{-1} g_{, X}\right)\left(1+\gamma^{-3} g_{, X}\right)},
\end{aligned}
$$

where $\chi$ is a general scalar field denoting either $\theta$ (minimal field) or $\Phi$ (conformal field). Because of the numerical facts

$$
\gamma=\mathcal{O}(10), g_{, X}=\mathcal{O}(1), \frac{g_{, X}^{\prime} \chi}{g_{, X}}=\mathcal{O}(1), \frac{f^{\prime} \chi}{f}=\mathcal{O}(1)
$$

we can safely set

$$
A \simeq 1, \quad B \simeq 1, \quad \frac{\dot{\gamma}}{H \gamma^{2}} \simeq-c_{s} s
$$

Thus,

$$
-\left(n_{s}-1\right)-4 \epsilon \simeq \frac{3}{2} g_{, X} c_{s} s-\frac{\dot{f}}{H f}=\frac{3}{2} g_{, X} c_{s} s+\frac{4 \dot{\chi}}{H \chi},
$$

where we have used the ansatz $f \propto \chi^{-4}$. As another numerical fact, we know that the first term in the final expression is small $\left(g_{, X}=\mathcal{O}(1), c_{s}=\mathcal{O}(0.1), s=\mathcal{O}(0.1)\right)$. In order to rewrite the second term in terms of observables (and $\chi / M_{\mathrm{Pl}}$ ), we can use the relation

$$
\left(\frac{\dot{\chi}}{H \chi}\right)^{2}=\frac{2 X}{H^{2} \chi^{2}}=\frac{2 \epsilon}{\gamma+g_{, X}} \cdot\left(\frac{M_{\mathrm{Pl}}}{\chi}\right)^{2} \simeq \frac{2 c_{s} \epsilon}{\left(\chi / M_{\mathrm{Pl}}\right)^{2}} .
$$

Therefore, for $\chi>0$ and $\dot{\chi}<0$,

$$
n_{s}-1 \simeq-4 \epsilon+\frac{\sqrt{32 c_{s} \epsilon}}{\chi / M_{\mathrm{Pl}}} .
$$

From the expression

$$
r=16 c_{s} \epsilon
$$


we obtain

$$
n_{s}-1 \simeq-\frac{r}{4 c_{s}}+\frac{\sqrt{2 r}}{\chi / M_{\mathrm{Pl}}} .
$$

Finally, by using the formula

$$
f_{N L} \simeq \frac{1}{3 c_{s}^{2}}
$$

we find

$$
n_{s}-1 \simeq-\frac{r \sqrt{3 f_{N L}}}{4}+\frac{\sqrt{2 r}}{\chi / M_{\mathrm{Pl}}}
$$

\section{Appendix C: Generalized Lyth Bound} (51):

The generalized Lyth bound is derived from 40 and

$$
r=\frac{8}{M_{\mathrm{Pl}}^{2}} \sqrt{\frac{\left(1+\gamma^{-1} g_{, X}\right)^{3}}{1+\gamma^{-3} g_{, X}}}\left(\frac{1}{H} \frac{d \chi}{d t}\right)^{2} .
$$

Thus, we obtain

$$
\begin{array}{r}
\chi(N=55)-\chi(N=0) \\
=\frac{M_{\mathrm{Pl}}}{2 \sqrt{2}} \int_{\chi_{0}}^{\chi_{55}}\left[\frac{1+\gamma^{-3} g_{, X}}{\left(1+\gamma^{-1} g_{, X}\right)^{3}}\right]^{1 / 4} \sqrt{r} d N \\
\simeq \frac{M_{\mathrm{Pl}}}{2 \sqrt{2}} \int_{\chi_{0}}^{\chi_{55}}\left(1-\frac{3}{4} \gamma^{-1} g_{, X}\right) \sqrt{r} d N,
\end{array}
$$

where $d N=-H d t$.

\section{Appendix D: Derivation of Relation (53)}

By using Eqs. (51), 46) and (19), we find

$$
\begin{aligned}
\frac{3\left(\gamma+g_{, X}\right)}{\epsilon} & =\frac{3 M_{\mathrm{Pl}}^{2} H^{2}}{X}=\frac{1}{f X}[\gamma-1+f V]+g_{, X} \\
& =\frac{2 \gamma^{2}}{\gamma^{2}-1}[\gamma-1+f V]+g_{, X} .
\end{aligned}
$$

Thus,

$$
f V=\frac{\gamma^{2}-1}{2 \gamma^{2}}\left[\frac{3\left(\gamma+g_{, X}\right)}{\epsilon}-g_{, X}\right]-\gamma+1 .
$$

From

$$
\gamma=\frac{1}{c_{s}} \sqrt{\frac{1+\gamma^{-1} g_{, X}}{1+\gamma^{-3} g_{, X}}}, \quad \epsilon=\frac{r}{16 c_{s}},
$$

we can rewrite Eq. (D2) as

$$
\begin{aligned}
f V & =\left[1-c_{s}^{2} \frac{1+\gamma^{-3} g_{, X}}{1+\gamma^{-1} g_{, X}}\right] \cdot\left[\frac{24}{r} \sqrt{\frac{\left(1+\gamma^{-1} g_{, X}\right)^{3}}{1+\gamma^{-3} g_{, X}}}-\frac{g, X}{2}\right] \\
& -\frac{1}{c_{s}} \sqrt{\frac{1+\gamma^{-1} g_{, X}}{1+\gamma^{-3} g_{, X}}}+1 .
\end{aligned}
$$

This result applies to conformal models. On the other hand, for minimal models we have

$f V=\left(1-c_{s}^{2}\right) \frac{24}{r}-\frac{1}{c_{s}}+1=\left(1-\frac{1}{3 f_{N L}}\right) \frac{24}{r}-\sqrt{3 f_{N L}}+1$.

This means that, for minimal models, the microscopic quantity $f V$ is expressed in terms of observables $r$ and $f_{N L}$.

Now, for $f_{N L}=40$, the numerical facts

$$
c_{s} \simeq \gamma^{-1} \simeq 0.1, \quad g_{, X}=\mathcal{O}(1)
$$

lead us to a rough approximation

$$
f V \simeq \frac{24}{r}
$$

for small $r$.

\section{Appendix E: Corrections of Order $\mathcal{O}\left(\gamma^{-1}\right)$}

\section{Non-Minimally Coupled Models}

In order to improve accuracy of the formula $\sqrt{52}$ we can include corrections first-order in $\gamma^{-1}$. The result is

$$
\begin{aligned}
n_{s}- & 1 \simeq-\frac{r \sqrt{3 f_{N L}}}{4}-\frac{f^{\prime} \Phi}{4 f} \cdot \frac{\sqrt{2 r}}{\Phi / M_{\mathrm{Pl}}} \times \\
& \left\{1+\frac{3 g_{, X}}{4 \sqrt{3 f_{N L}}} \cdot\left[\frac{g^{\prime} \Phi}{2 g_{, X}} \cdot\left(-\frac{4 f}{f^{\prime} \Phi}\right)-1\right]\right\} \\
& -\frac{3 g_{, X} s}{2 \sqrt{3 f_{N L}}} .
\end{aligned}
$$

Here, we have used

$$
\begin{aligned}
B & \simeq 1-\frac{3}{2} \gamma^{-1} g_{, X} \cdot \frac{g_{, X}^{\prime} \Phi}{g_{, X}} \cdot \frac{f}{f^{\prime} \Phi} \\
c_{s} & \simeq \gamma^{-1}\left(1+\frac{1}{2} \gamma^{-1} g_{, X}\right) \\
\left(\frac{\dot{\Phi}}{H \Phi}\right)^{2} & \simeq \frac{2 c_{s} \epsilon}{\left(\Phi / M_{\mathrm{Pl}}\right)^{2}} \times\left(1-\frac{3}{2} \gamma^{-1} g_{, X}\right) .
\end{aligned}
$$

By using $f \propto \Phi^{-4}$ and $g_{, X}=\tan ^{2}\left(\Phi / \sqrt{6} M_{\mathrm{Pl}}\right)$, we obtain

$$
\begin{aligned}
n_{s}- & 1 \simeq-\frac{r \sqrt{3 f_{N L}}}{4}+\frac{\sqrt{2 r}}{\Phi / M_{\mathrm{Pl}}} \times \\
& \left\{1+\frac{3 g_{, X}}{4 \sqrt{3 f_{N L}}}\left[\frac{\left(2 \Phi / \sqrt{6} M_{\mathrm{Pl}}\right)}{\sin \left(2 \Phi / \sqrt{6} M_{\mathrm{Pl}}\right)}-1\right]\right\} \\
& -\frac{3 g_{, X} s}{2 \sqrt{3 f_{N L}}} .
\end{aligned}
$$




\section{Minimally Coupled Models}

The corresponding relation for minimially coupled models can be obtained by setting $g_{, X}=0$. However, we have to be careful about the last term in $A$ shown in (B2). Then we obtain

$$
-\left(n_{s}-1\right)-4 \epsilon=\frac{2 \dot{\gamma}}{H \gamma^{3}} \cdot \frac{1}{1-\gamma^{-2}}-\frac{\dot{f}}{H f} .
$$

This leads to the following approximate relation

$$
n_{s}-1 \simeq-\frac{r \sqrt{3 f_{N L}}}{4}+\frac{\sqrt{2 r}}{\theta / M_{\mathrm{Pl}}}+\frac{2 s}{3 f_{N L}} .
$$

Interestingly enough, minimally coupled models have red spectra $\left(n_{s}<1\right)$ in the limit $r \rightarrow 0$ since $s$ is negative. Here, we have used numerical values $20<f_{N L}<40$, $|s|=\mathcal{O}(1)$ and $\theta / M_{\mathrm{Pl}}=\mathcal{O}(1)$.
[1] A. H. Guth, Phys. Rev. D 23, 347 (1981).

[2] A. D. Linde, Phys. Lett. B 108, 389 (1982).

[3] A. Albrecht and P. J. Steinhardt, Phys. Rev. Lett. 48, 1220 (1982).

[4] E. Komatsu et al. [WMAP Collaboration], Astrophys. J. Suppl. 180, 330 (2009) arXiv:0803.0547 [astro-ph]].

[5] R. H. Brandenberger, Lect. Notes Phys. 738, 393 (2008) arXiv:hep-th/0701111.

[6] G. R. Dvali and S. H. H. Tye, Phys. Lett. B 450, 72 (1999) arXiv:hep-ph/9812483.

[7] S. H. S. Alexander, Phys. Rev. D 65, 023507 (2002) arXiv:hep-th/0105032.

[8] G. R. Dvali, Q. Shafi and S. Solganik, arXiv:hepth/0105203

[9] C. P. Burgess, M. Majumdar, D. Nolte, F. Quevedo, G. Rajesh and R. J. Zhang, JHEP 0107, 047 (2001) arXiv:hep-th/0105204.

[10] J. H. Brodie and D. A. Easson, JCAP 0312, 004 (2003) arXiv:hep-th/0301138.

[11] S. Kachru, R. Kallosh, A. Linde, J. M. Maldacena, L. P. McAllister and S. P. Trivedi, JCAP 0310, 013 (2003) arXiv:hep-th/0308055.

[12] L. McAllister and E. Silverstein, Gen. Rel. Grav. 40, 565 (2008) arXiv:0710.2951 [hep-th]].

[13] D. Baumann and L. McAllister, arXiv:0901.0265 [hepth].

[14] C. Armendariz-Picon, T. Damour and V. F. Mukhanov, Phys. Lett. B 458, 209 (1999) arXiv:hep-th/9904075.

[15] N. Arkani-Hamed, P. Creminelli, S. Mukohyama and M. Zaldarriaga, JCAP 0404, 001 (2004) arXiv:hepth/0312100.

[16] E. Silverstein and D. Tong, Phys. Rev. D 70, 103505 (2004) arXiv:hep-th/0310221.

[17] J. Garriga and V. F. Mukhanov, Phys. Lett. B 458, 219 (1999).

[18] M. Alishahiha, E. Silverstein and D. Tong, Phys. Rev. D 70, 123505 (2004) arXiv:hep-th/0404084.

[19] X. Chen, M. x. Huang, S. Kachru and G. Shiu, JCAP 0701, 002 (2007) arXiv:hep-th/0605045.

[20] N. D. Birrell and P. C. W. Davies, Cambridge, Uk: Univ. Pr. ( 1982) $340 p$.

[21] N. Seiberg and E. Witten, JHEP 9904, 017 (1999) arXiv:hep-th/9903224.

[22] A. Fotopoulos and A. A. Tseytlin, JHEP 0212, 001 (2002) arXiv:hep-th/0211101.
[23] C. P. Burgess, H. M. Lee and M. Trott, JHEP 0909, 103 (2009) arXiv:0902.4465 [hep-ph]].

[24] I. R. Klebanov and M. J. Strassler, JHEP 0008, 052 (2000) arXiv:hep-th/0007191.

[25] X. Chen, S. Sarangi, S. H. Henry Tye and J. Xu, JCAP 0611, 015 (2006) arXiv:hep-th/0608082.

[26] D. Baumann and L. McAllister, Phys. Rev. D 75, 123508 (2007) arXiv:hep-th/0610285.

[27] H. V. Peiris, D. Baumann, B. Friedman and A. Cooray, Phys. Rev. D 76, 103517 (2007) arXiv:0706.1240 [astro$\mathrm{ph}]$.

[28] D. A. Easson, R. Gregory, D. F. Mota, G. Tasinato and I. Zavala, JCAP 0802, 010 (2008) arXiv:0709.2666 [hepth]].

[29] R. Bean, X. Chen, H. Peiris and J. Xu, Phys. Rev. D 77, 023527 (2008) arXiv:0710.1812 [hep-th]].

[30] L. Leblond and S. Shandera, JCAP 0808, 007 (2008) arXiv:0802.2290 [hep-th]].

[31] M. Becker, L. Leblond and S. E. Shandera, Phys. Rev. D 76, 123516 (2007) arXiv:0709.1170 [hep-th]].

[32] E. Silverstein and A. Westphal, Phys. Rev. D 78, 106003 (2008) arXiv:0803.3085 [hep-th]].

[33] L. McAllister, E. Silverstein and A. Westphal, arXiv:0808.0706 [hep-th].

[34] A. Avgoustidis and I. Zavala, JCAP 0901, 045 (2009) arXiv:0810.5001 [hep-th]].

[35] A. A. Starobinsky, Pis'ma Astron. Zh. 7, 67 (1981).

[36] T. Futamase, T. Rothman and R. Matzner, Phys. Rev. D 39, 405 (1989).

[37] N. Makino and M. Sasaki, Prog. Theor. Phys. 86, 103 (1991).

[38] P. Creminelli, JCAP 0310, 003 (2003) arXiv:astro$\mathrm{ph} / 0306122$.

[39] R. Bean, S. E. Shandera, S. H. Henry Tye and J. Xu, JCAP 0705, 004 (2007) arXiv:hep-th/0702107.

[40] J. M. Maldacena, JHEP 0305, 013 (2003) arXiv:astro$\mathrm{ph} / 0210603$.

[41] A. Gangui, F. Lucchin, S. Matarrese and S. Mollerach, Astrophys. J. 430, 447 (1994) arXiv:astro-ph/9312033.

[42] E. Komatsu and D. N. Spergel, Phys. Rev. D 63, 063002 (2001) arXiv:astro-ph/0005036.

[43] D. Babich, P. Creminelli and M. Zaldarriaga, JCAP 0408, 009 (2004) arXiv:astro-ph/0405356.

[44] J. R. Fergusson and E. P. S. Shellard, arXiv:0812.3413 [astro-ph]. 
[45] D. A. Easson and R. Gregory, Phys. Rev. D 80, 083518 (2009) arXiv:0902.1798 [hep-th]].

[46] R. Easther and W. H. Kinney, Phys. Rev. D 67, 043511 (2003) arXiv:astro-ph/0210345.

[47] B. A. Powell and W. H. Kinney, JCAP 0708, 006 (2007) arXiv:0706.1982 [astro-ph]].

[48] L. Lorenz, J. Martin and C. Ringeval, Phys. Rev. D 78, 063543 (2008) arXiv:0807.2414 [astro-ph]].

[49] L. Lorenz, J. Martin and C. Ringeval, Phys. Rev. D 78, 083513 (2008) arXiv:0807.3037 [astro-ph]].

[50] N. Agarwal and R. Bean, Phys. Rev. D 79, 023503 (2009)
arXiv:0809.2798 [astro-ph]].

[51] B. A. Powell, K. Tzirakis and W. H. Kinney, JCAP 0904, 019 (2009) arXiv:0812.1797 [astro-ph]].

[52] L. Senatore, K. M. Smith and M. Zaldarriaga, arXiv:0905.3746 [astro-ph.CO].

[53] D. H. Lyth, Phys. Rev. Lett. 78, 1861 (1997) arXiv:hep$\mathrm{ph} / 9606387$.

[54] D. Babich and M. Zaldarriaga, Phys. Rev. D 70, 083005 (2004) arXiv:astro-ph/0408455. 